\title{
The Capacity Optimization of Wind-Photovoltaic-Thermal Energy Storage Hybrid Power System
}

\author{
Jingli $\mathrm{Li}^{1}{ }^{1}$,Wannian $\mathrm{Qi}^{1}{ }^{1}$, Jun $\mathrm{Yang}^{2}, \mathrm{Yi} \mathrm{He}^{3}$, Jingru $\mathrm{Luo}^{4}$, and $\mathrm{Su} \mathrm{Guo}^{3, *}$ \\ ${ }^{1}$ Qinghai Golmud Luneng Energy Co., Ltd (Ducheng Weiye Group Co. Ltd), Qinghai, China \\ ${ }^{2}$ Qinghai Electric Power Research Institute, Qinghai, China \\ ${ }^{3}$ College of Energy and Electrical Engineering Hohai University,Nanjing, Jiangsu, China \\ ${ }^{4}$ East China Yixing Pumped Storage Power Co., Ltd. Jiangsu, China
}

\begin{abstract}
This paper proposes a Wind-Photovoltaic-Thermal Energy Storage hybrid power system with an electric heater. The proposed system consists of wind subsystem, photovoltaic subsystem, electric heater, thermal energy storage and steam turbine unit. The electric heater is used to convert the redundant electricity from wind or photovoltaic subsystem into heat, which is stored in thermal energy storage. When the system output is less than the load demand, thermal energy storage system releases heat to generate electricity. In this paper, the optimal objective is to minimize the levelized cost of energy and maximize the utilization rates of renewable energy and transmission channel. The fitness function is compiled according to the scheduling strategy, and the capacity optimization problem is solved by particle swarm optimization algorithm in MATLAB. The case analysis show that the proposed system can effectively increase the utilization rate of renewable energy and transmission channel.
\end{abstract}

\section{Introduction}

With the development of science and technology, energy and environment problem have become the two most popular topics. Wind power and photovoltaic power (PV) are the most widely applied forms of renewable energy generation. However, because of the intermittency and instability of wind and solar resources, the dispatchability and flexibility of wind or PV system are limited. Moreover, the fluctuation of wind power output even may influence the grid stability during integration process[1]. Solar resource is negatively correlated with wind resource, so the output of wind power and solar power are generally complementary[2]. Therefore, the researches of windsolar hybrid system are very popular in recent years.

Yao et al researched the capacity optimization of wind-PV system without energy storage, where PV modules are constructed in the wind farm[3]. Muhammad et al analysed the tech-economy of a hybrid wind-PVbattery system with genetic algorithm, which concentrates on the loss of power supply probability effect on cost of energy[4]. Wu et al utilized the battery-ultra capacitor hybrid storage units to improve the reliability of wind-PV complementary system and proposes the capacity optimization model of hybrid storage unit[5]. Dai et al utilized the Computer Aided Design (CAD) method to design the optimum ratio of wind-PV complementary system[6]. From above references, it can be seen that conventional wind-PV system utilizes battery as energy storage, but battery storage has many disadvantages such as strict charging and discharging limitation, expensive investment and short cycle life.

Concentrated Solar Power (CSP) is one of the most promising technologies, which consists of solar field, thermal energy storage (TES) and power block (PB). CSP plant with TES can be dispatched as a conventional power plant. Therefore, many scholars proposed to integrate wind farm and/or PV with CSP to improve the reliability and dispatchability of power. Yang et al utilized electric heater to mitigate wind power fluctuation and reduce wind curtailment, and proposes the day-ahead schedule for wind-CSP hybrid system with mixed integer linear programming (MILP) model[7]. Cui et al developed the optimized capacity configuration model of wind-CSP system, which is based on the time-sharing energy complementary characteristics between wind and CSP output[8]. Petrollese et al researched the optimum ratio of PV-CSP hybrid system and evaluate the effect of load demand durations and allowed unmet load fraction on the levelized cost of energy[9]. Cui et al proposed the schedule strategy of wind-PV-CSP system, but each subsystem are independent and has no direct energy connection[10].

The development of CSP plant has obstacles such as the huge investment caused by solar field, which accounts for approximately $50 \%$ of total cost. Therefore, this paper proposes a wind-PV-TES hybrid system with an electric heater. The proposed system only utilizes TES and power block to adjust system output, which omits the expensive solar field of CSP. If the output of wind and PV are greater than load demand, the electric heater converts the

\footnotetext{
* Corresponding author: guosu81@126.com
} 
redundant electricity from wind or PV subsystem into thermal energy stored in TES. Otherwise, TES releases thermal energy into the steam turbine units to generate electricity for supplying power shortage.

In this paper, the configuration of the proposed system is introduced in Section 2. The simulation models of each component are introduced in Section 3. The fitness and constraint of optimization are introduced in Section 4. The scheduling strategy of the proposed system is introduced in Section 5.The comparison between the proposed system and wind-PV system without energy storage is shown in Section 6. Conclusions are shown in Section 7.

\section{The configuration of hybrid system}

The configuration of the hybrid system is shown as Fig. 1. The wind-PV-TES system in this research consists of a wind subsystem, a PV subsystem, a TES subsystem with power block and an electric heater.

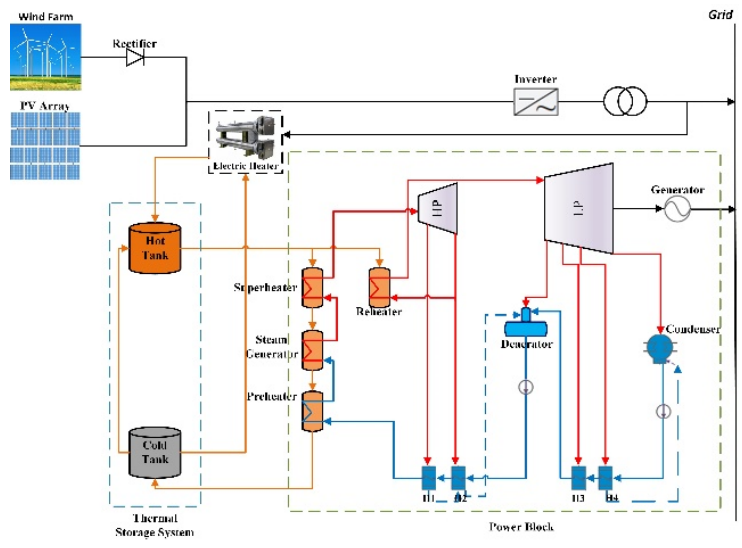

Fig. 1. The configuration of the Wind-PV-Thermal Energy Storage hybrid system with an Electric Heater

The electricity generated by wind subsystem need to be rectified and then share a direct current (DC) bus with PV subsystem. The DC electricity is converted to alternating current (AC) by the inverter and boosted by the boost substation, then integrated to the power grid. If the power output of wind and PV subsystems surplus the demand of the power grid, the redundant electricity will be converted into thermal energy by the electric heater and stored in TES. When the output of wind and PV subsystems are less than the demand of the power grid, the TES system will release thermal energy into the power block to generate for supplying the electricity shortage. The TES can not only mitigate the fluctuation of wind and PV output, reduce the wind curtailment and solar curtailment, but also adjust the period of the system output, so that effectively increase the utilization rate of transmission channel and renewable energy.

The Electric Heater (EH) in this system adopt $4160 \mathrm{~V}$ medium-voltage system. Compared with the traditional $480 \mathrm{~V}$ low-voltage system, the medium-voltage EH system can not only effectively reduce the energy loss in the heating process, but also reduce the cost of installation, operation and maintenance, so that effectively reduce the investment cost of the system.

\section{Mathematical modelling of system components}

\subsection{Wind model}

The power output generated by wind turbine depending on actual wind speed and its power output curve can be expressed as ${ }^{[1]}$ :

$$
\begin{gathered}
P_{\mathrm{w}}=\left\{\begin{array}{l}
0 u<u_{\mathrm{ci}} \text { or } u>u_{\mathrm{co}} \\
f(u) \quad u_{\mathrm{ci}} \leq u \leq u_{\mathrm{r}} \\
P_{\mathrm{r}} \quad u_{\mathrm{r}} \leq u \leq u_{\mathrm{co}}
\end{array}\right. \\
f(u)=\frac{u_{\mathrm{ci}}^{3}}{u_{\mathrm{r}}^{3}-u_{\mathrm{ci}}^{3}} \cdot P_{\mathrm{r}}
\end{gathered}
$$

where $u$ is actual wind speed, $u_{\text {ci }}, u_{\text {co }}$ and $u_{\mathrm{r}}$ are cutin, cut-off and rated wind speed of wind turbines, $P_{\mathrm{w}}$ and $P_{\mathrm{r}}$ are actual and rated power output of wind turbines.

\subsection{Photovoltaic model}

The PV module power output depending on solar radiation and environment temperature is expressed as ${ }^{[13]}$ :

$$
P_{\mathrm{pv}}=P_{\mathrm{stc}} \cdot \frac{s}{S_{\mathrm{stc}}}\left(1+\varepsilon \cdot\left(T-T_{\mathrm{stc}}\right)\right)
$$

where $P_{\mathrm{pv}}$ and $P_{\mathrm{stc}}$ are actual PV module power output and maximum power output under standard test conditions. $S$ and $S_{\text {stc }}$ are actual solar radiation and standard test radiation. $T$ and $T_{\text {stc }}$ are actual cell temperature of PV module and standard test temperature. $\varepsilon$ is the temperature coefficient of power.

\subsection{TES model}

Assuming that the storage and discharge power of TES is constant in period $t$, the heat storage of TES after the storage and discharge process is shown as ${ }^{[14]}$ :

$$
\begin{aligned}
& W_{\mathrm{TES}}^{t+1}=W_{\mathrm{TES}}^{t} \cdot\left(1-\delta_{\mathrm{TES}}\right)+H_{\mathrm{TES} . c}^{t} \cdot \eta_{\mathrm{TES} . c} \cdot \Delta t \\
& W_{\mathrm{TES}}^{t+1}=W_{\mathrm{TES}}^{t} \cdot\left(1-\delta_{\mathrm{TES}}\right)+\frac{H_{\mathrm{TES} . \mathrm{d}}^{t}}{\eta_{\mathrm{TES} .} \cdot \Delta t}
\end{aligned}
$$

where $W_{\text {TES }}^{t+1}$ and $W_{\text {TES }}^{t}$ are the heat storage of TES in period $t+1$ and $t, H_{\mathrm{TES} . c}^{t}$ and $H_{\mathrm{TES} . \mathrm{d}}^{t}$ are the storage and discharge power per unit time respectively, $\eta_{\text {TES.c }}$ and $\eta_{\text {TES.d }}$ are the storage and discharge efficiency separately, $\delta_{\mathrm{TES}}$ is the rate of heat loss, $\Delta t$ is time interval.

\subsection{Power block model}

The power output of power block is shown as:

$$
P_{\text {out }}^{\mathrm{PB}}=\eta_{\mathrm{e}} \cdot P_{\text {in }}^{\mathrm{PB}}
$$

where $P_{\mathrm{out}}^{\mathrm{PB}}$ and $P_{\mathrm{in}}^{\mathrm{PB}}$ are the power output and input of power block respectively, $\eta_{\mathrm{e}}$ is power generation efficiency.

\subsection{Electric model}

The power output of electric heater is shown as:

$$
P_{\text {out }}^{\mathrm{EH}}=\eta_{\mathrm{H}} \cdot P_{\text {in }}^{\mathrm{EH}}
$$

where $P_{\mathrm{out}}^{\mathrm{EH}}$ and $P_{\mathrm{in}}^{\mathrm{EH}}$ are the power output and input of electric heater respectively, $\eta_{\mathrm{H}}$ is electric heater efficiency. 


\section{Fitness function}

The optimal objective of the proposed system is to minimize the levelized cost of energy (LCOE) and maximize the utilization rates of renewable energy and transmission channel. Moreover, LCOE is used for evaluating the economy of the hybrid system, the utilization rates of transmission channel and renewable energy are used for evaluating the reliability of the system. The optimal variables are the capacities of wind, PV, TES and $\mathrm{PB}$.

The LCOE is a measure of the mean energy production cost over the plant lifetime. It is a very useful indicator because it allows a comparison between various energy technologies even when the rated power and investment costs are different ${ }^{[9]}$. The LCOE of the windPV-TES hybrid system with an electric heater is shown as:

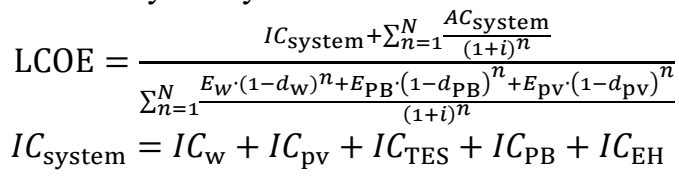

$A C_{\text {system }}=A C_{\mathrm{w}}+A C_{\mathrm{pv}}+A C_{\mathrm{TES}}+A C_{\mathrm{PB}}+A C_{\mathrm{EH}}$

where $I C_{\text {system }}$ is the initial cost of the hybrid system, which mainly includes the cost of equipment and construction. $A C_{\text {system }}$ is the annual cost of the hybrid system, which mainly includes the cost of operation and maintenance. $d_{\mathrm{w}}, d_{\mathrm{pv}}$ and $d_{\mathrm{PB}}$ are the annual degradation of wind, PV and PB subsystem respectively. $E_{\mathrm{w}}, E_{\mathrm{pv}}$ and $E_{\mathrm{PB}}$ are the first-year electricity output of wind, PV and PB subsystem respectively. $i$ is the discount rate, and $\mathrm{N}$ is the expected lifetime of the system.

The channel utilization rate of $R_{c h}$ refers to the ratio of total on-grid electricity to annual channel capacity, which is shown as follow.

$$
R_{\mathrm{ch}}=\frac{\sum_{i}^{8760} E_{\mathrm{w} . i}+E_{\mathrm{pv} . i}+E_{\mathrm{PB} . i}}{E_{\mathrm{ch}} * 8760}
$$

where $E_{\mathrm{w} . i}, E_{\mathrm{pv} . i}$ and $E_{\mathrm{PB} . i}$ are the on-grid electricity of wind, $\mathrm{PV}$ and $\mathrm{PB}$ subsystems during period $t . E_{\mathrm{ch}}$ is the channel capacity. Time interval is 1 hour.

The utilization rate of renewable energy $R_{r e}$ refers to the ratio between the total on-grid electricity and the total power output of wind and PV subsystems, which is shown as:

$$
R_{\mathrm{re}}=\frac{\sum_{i}^{8760} E_{\mathrm{w} . i}+E_{\mathrm{pv} . i}+E_{\mathrm{PB} . i}}{\sum_{i}^{8760} P_{\mathrm{w} . i}+P_{\mathrm{pv} . i}}
$$

where $P_{\mathrm{w} . i}$ and $P_{\mathrm{pv} . i}$ are the power output of wind and PV subsystems during period $t$, respectively.

The fitness function of this optimization problem is shown as:

$$
\text { fitness }=\omega_{1} * L C O E+\frac{\omega_{2}}{R_{\mathrm{ch}}}+\frac{\omega_{3}}{R_{\mathrm{re}}}
$$

where $\omega_{1}, \omega_{2}, \omega_{3}$ are the optimization weight of LCOE, $R_{\mathrm{ch}}$ and $R_{\text {re }}$, respectively. The optimization weight can be adjusted according to different optimization requirements to obtain the optimal solution suitable for different scenarios.

\section{Scheduling strategy}

The scheduling strategy of the hybrid system is shown as Fig. 2. In the scheduling strategy, $p w(i), p v(i)$ are the power output of wind and PV subsystems during period $i$, respectively. $c_{-}$load is the capacity of transmission channel. $g w(i), g v(i)$ and $g p(i)$ are the electricity integrated to the power grid of wind, PV and PB subsystem respectively. $\operatorname{dp}(i)$ is the difference between the output of wind and PV and transmission channel capacity.

If $d p(i)>0$, the output of wind and PV exceeds the channel capacity limitation, the redundant electricity is converted to thermal energy by the EH and then stored in TES. If the redundant electricity exceed the maximum storage capacity of TES, there will be wind and solar curtailment. Because the electricity price of wind power is higher than $\mathrm{PV}$, this paper assumes that the wind power will be integrated to the power grid firstly. If $d p(i)<0$, the output of wind and PV is less than the channel capacity, TES will release thermal energy into PB to generate for supplying the shortage in this situation. $g p$ is used to judge whether the turbine needs to preheat. If the turbine unit operates during Period $i-1$, that is to say $g p(i-$ 1) $>0$, the steam turbine unit does not need preheating during Period $i$, vice versa. $\eta$ is the generation efficiency of $\mathrm{PB}$, pre is the preheating coefficient. The initial capacity of TES is set as half of the total capacity of TES. The capacity of TES, $m s$, is always satisfied the following relationship, in which $\mathrm{ms}_{-} \min$ and $\mathrm{ms}_{-} \max$ are the minimum and maximum effective values of TES capacity, respectively.

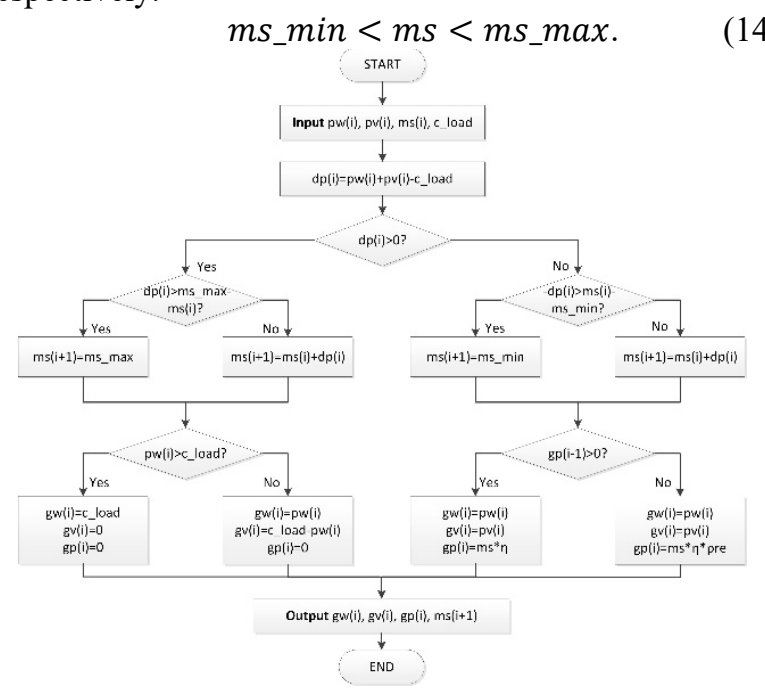

Fig. 2. The flow chart of scheduling strategy

\section{Case study}

\subsection{Data preparation}

In this paper, a wind-PV complementary system is proposed to make full use of $100 \mathrm{MW}$ transmission channel capacity in a specific area. According to the wind resource data provided by the local wind tower and the solar radiation data provided by Solargis, the wind and PV outputs are calculated by the mathematical models.

The comprehensive assessment of wind resource provided by local wind towers is shown as Fig. 3. The yearly average wind speed is $6.43 \mathrm{~m} / \mathrm{s}$, the yearly average 
wind power density is $262.9 \mathrm{~W} / \mathrm{m}^{2}$, the wind resource in this area is relatively rich.

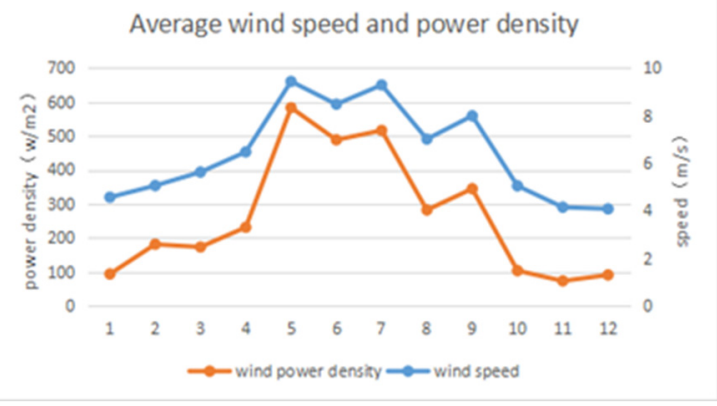

Fig.3. The monthly average wind speed and power density

The comprehensive assessment of solar radiation resource provided by Solargis is shown as Fig. 4. The yearly-total average global horizontal irradiance (GHI) is $2091.8 \mathrm{~W} / \mathrm{m}^{2}$, the yearly-total average direct normal irradiance (DNI) is $930.7 \mathrm{~W} / \mathrm{m}^{2}$, the yearly-total average diffuse horizontal irradiation (DHI) is $1161.1 \mathrm{~W} / \mathrm{m}^{2}$, the solar resource in this area is also rich. The PV subsystem can operate by using DNI and DHI.

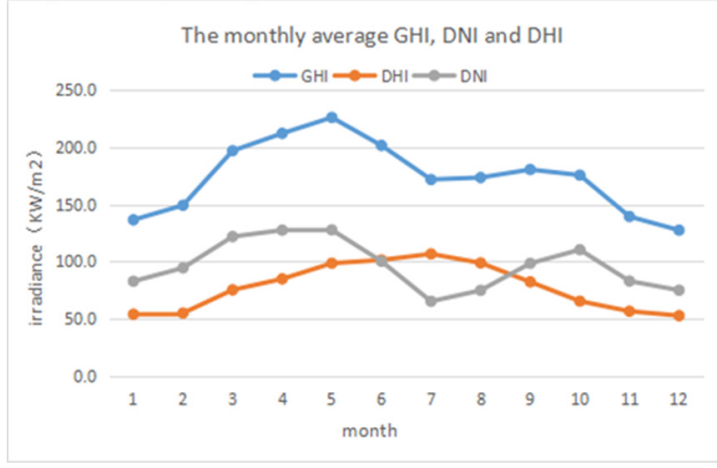

Fig.4. The monthly average GHI, DNI and DHI

\subsection{Power output prediction}

According to the wind resource and mathematical model, the power output curve of $1 \mathrm{MW}$ wind capacity is calculated and shown as Fig. 5.

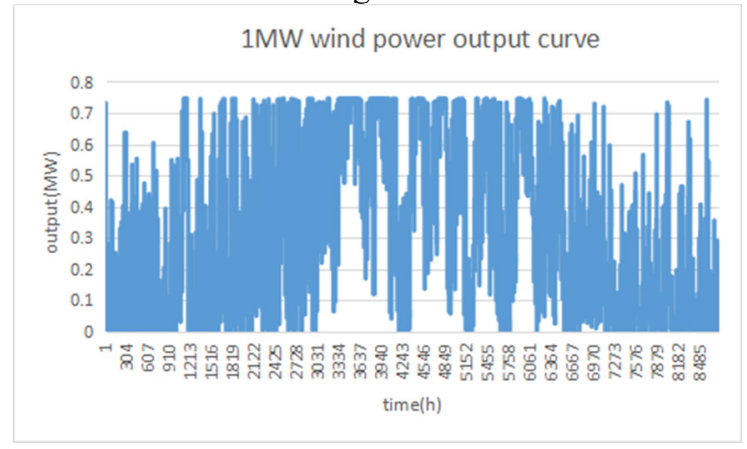

Fig.5. The power output of 1MW wind capacity

According to the solar radiation resource and relative mathematical model, the power output curve of 1MW PV capacity is calculated and shown as Fig. 6.

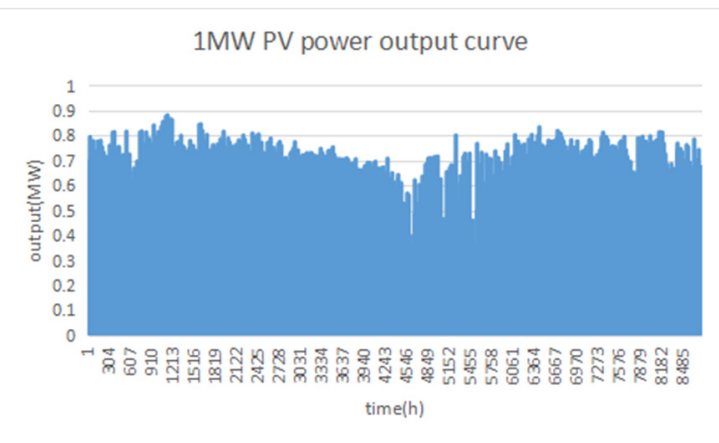

Fig.6. The power output curve of 1MW PV capacity

\subsection{Optimization results}

The economic parameters which is used to calculate LCOE are shown as Table 1.

Table 1 The economic parameters

\begin{tabular}{|c|c|c|c|c|c|}
\hline & wind & PV & TES & PB & EH \\
\hline IC: $\$ \mathbf{k W}$ & 1695 & 1040 & 68 & 923 & 528655 \\
\hline $\mathbf{A C}: \$ \mathbf{k W}$ & 51 & 9 & 10 & 12 & 12550 \\
\hline $\mathbf{d}$ & 0 & $0.8 \%$ & 0 & 0 & 0 \\
\hline Price:\$/kWh & 0.08 & 0.06 & 0 & 0.18 & 0 \\
\hline
\end{tabular}

(Remarks: economic parameters refer to SAM software, the electricity price of PB refer to CSP plants.)

The parameters of PSO algorithm is determined as follows. Initial population $\mathrm{N}=50$, the learning factor $\mathrm{C} 1=$ $\mathrm{C} 2=2.0$, the inertia weight $\mathrm{w}=0.5$, the search space dimension $\mathrm{D}=4$, and the iteration step $\mathrm{M}=20$. The optimization results based on different optimization weights are shown in Table 2.

Table 2 The optimization results

\begin{tabular}{|c|c|c|c|c|c|c|}
\hline wind & PV & TES & PB & LCOE & $\boldsymbol{R}_{c h}$ & $R_{r e}$ \\
\hline \multicolumn{7}{|c|}{$\mathrm{w} 1=0.2, \quad \mathrm{w} 2=0.6, \quad \mathrm{w} 3=0.2$} \\
\hline 162 & 200 & 9.4 & 137 & 1.58 & 0.73 & 0.76 \\
\hline \multicolumn{7}{|c|}{$\mathrm{w} 1=0.2, \quad \mathrm{w} 2=0.5, \quad \mathrm{w} 3=0.3$} \\
\hline 139 & 200 & 9.3 & 88 & 1.45 & 0.70 & 0.79 \\
\hline \multicolumn{7}{|c|}{$\mathrm{w} 1=0.2, \quad \mathrm{w} 2=0.4, \quad \mathrm{w} 3=0.4$} \\
\hline 128 & 161 & 9.6 & 54 & 1.37 & 0.66 & 0.85 \\
\hline \multicolumn{7}{|c|}{$\mathrm{w} 1=0.3, \quad \mathrm{w} 2=0.4, \quad \mathrm{w} 3=0.3$} \\
\hline 135 & 153 & 8.5 & 45 & 1.35 & 0.65 & 0.85 \\
\hline \multicolumn{7}{|c|}{$\mathrm{w} 1=0.4, \quad \mathrm{w} 2=0.3, \quad \mathrm{w} 3=0.3$} \\
\hline 122 & 125 & 2.0 & 53 & 1.30 & 0.60 & 0.88 \\
\hline
\end{tabular}

According to the optimization results, when the optimization weight of $R_{c h}, \mathrm{w} 2$, is large, the capacity of TES and PB is also large. When the optimization weight of LCOE w1 is large, the capacity of TES and PB is very little. When the capacity of wind and PV is small, the utilization rate of renewable energy $R_{r e}$ will be large, because the channel capacity is enough to consume the most of electricity generated by wind and PV subsystems. 
The main role of TES in the wind-PV-TES hybrid system is mitigating the fluctuation of power output and adjusting the supplying period. Therefore, three groups of optimization results with larger optimization weight of $R_{c h}$ and $R_{r e}$ are selected to compare with wind-PV system without energy storage, the specific comparison is shown as Table 3.

Table 3 The comparison between wind-PV-TES system(1) and wind-PV system(2)

\begin{tabular}{|c|c|c|c|c|c|c|c|}
\hline & wind & PV & TES & PB & LCOE & $\boldsymbol{R}_{c h}$ & $\boldsymbol{R}_{r e}$ \\
\hline \multicolumn{8}{|c|}{$w 1=0.2, \quad w 2=0.6, \quad w 3=0.2$} \\
\hline (1) & 162 & 200 & 9.4 & 137 & 1.58 & 0.73 & 0.76 \\
\hline (2) & 162 & 200 & 0 & 0 & 1.44 & 0.67 & 0.70 \\
\hline \multicolumn{8}{|c|}{$\mathrm{w} 1=0.2, \quad w 2=0.5, \quad w 3=0.3$} \\
\hline (1) & 139 & 200 & 9.3 & 88 & 1.45 & 0.70 & 0.79 \\
\hline (2) & 139 & 200 & 0 & 0 & 1.37 & 0.65 & 0.73 \\
\hline \multicolumn{8}{|c|}{$\mathrm{w} 1=0.2, \quad w 2=0.4, \quad w 3=0.4$} \\
\hline (1) & 128 & 161 & 54 & 9.6 & 1.37 & 0.66 & 0.85 \\
\hline (2) & 128 & 161 & 0 & 0 & 1.25 & 0.63 & 0.81 \\
\hline
\end{tabular}

As shown in Table 3, Compared to wind-PV system, the LCOE of wind-PV-TES system increases because of the investment cost of EH, TES and $\mathrm{PB}$, The increase range are 9.7\%, 5.8\% and 9.6\% respectively for different optimization weight. However, it is predictable that the cost of TES and EH will decrease dramatically in the future. Moreover, the PB can utilize the steam turbine unit of small conventional coal-fired power plants planned to rectify or shut down, which can greatly reduce the investment cost of the proposed system. Therefore, the proposed system has a good prospect of economical efficiency.

Through adding EH, TES and PB to the wind-PV system, the utilization rates of transmission channel and renewable energy have been significantly increased. Compared to wind-PV system, $R_{c h}$ increases by $6 \%, 5 \%$ and $3 \%$ respectively for different optimization weight, $R_{r e}$ increases by $6 \%, 6 \%$ and $4 \%$ respectively. Therefore, TES system can effectively increase the utilization rate of transmission channel and renewable energy. Moreover, the electricity quality generated by PB is better than wind or PV subsystem, and it will not cause voltage fluctuation and frequency flicker of the power grid, which is gridfriendly. Therefore, the TES and PB can effectively increase the reliability of wind-PV system.

The theoretical output of wind farm and PV plant, the actual on-grid power of wind-PV-TES system and the actual on-grid power of wind-PV system on the representative days of each season in a year is shown as from Fig. 7 to Fig. 10.

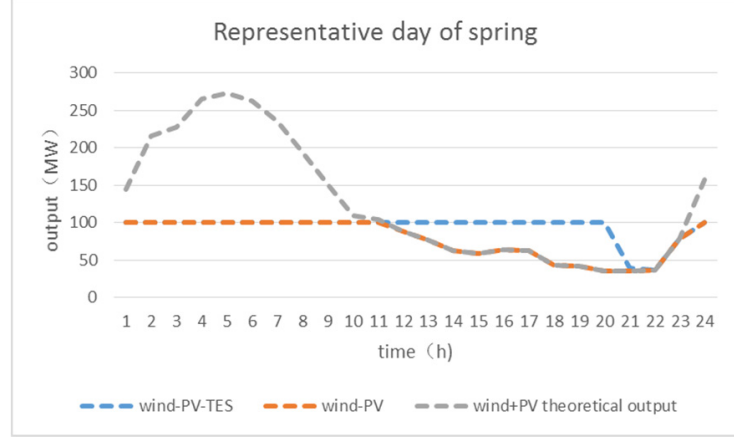

Fig. 7 The comparison on spring representative day

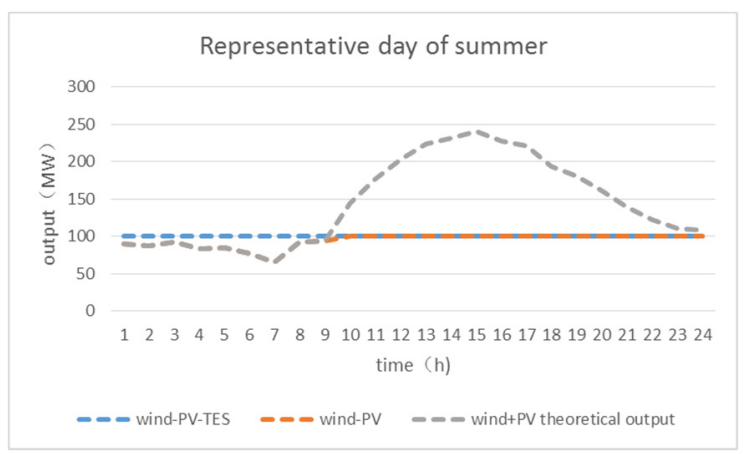

Fig. 8 The comparison on summer representative day

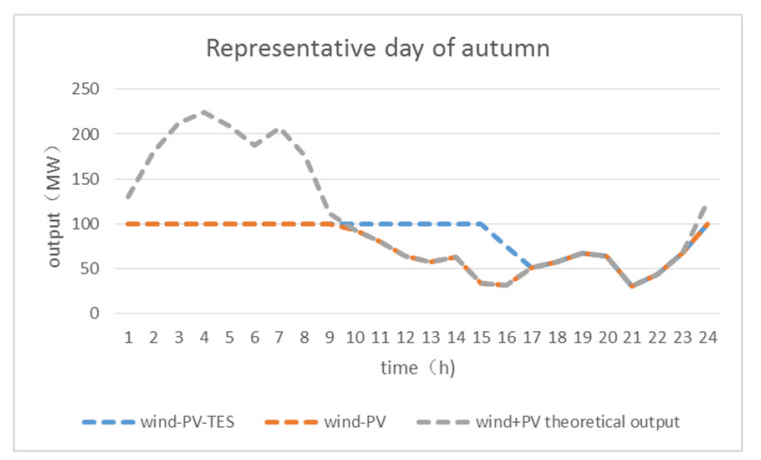

Fig. 9 The comparison on autumn representative day

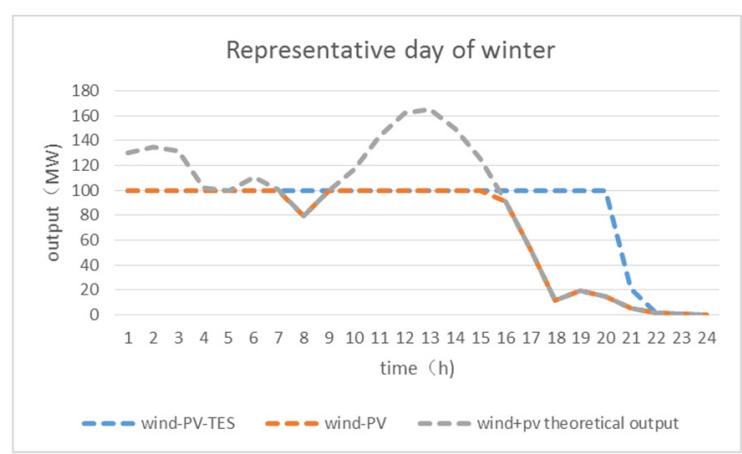

Fig. 10 The comparison on winter representative day

From Fig. 7-Fig. 10, compared with the wind-PV system, the wind-PV-TES hybrid system can meet the full load demand of the transmission channel in most of the time. The wind-PV-TES hybrid system can effectively increase the utilization rate of transmission channel and renewable energy. 


\section{Conclusion}

This paper proposes a wind-PV-TES hybrid system, and introduces the configuration and scheduling strategy of the system. The optimization objective of capacity configuration is to minimize LCOE and maximize the utilization rates of transmission channel and renewable energy. The optimal capacity ratio for different optimization weight is calculated by PSO algorithm. In case study, this paper compares wind-PV-TES hybrid system with wind-PV system without energy storage in economy and reliability. The results show that the proposed system in this paper can effectively adjust the power output and increase the reliability of wind-PV complementary system, which highlight the feasibility and superiority of wind-PV-TES hybrid system.

Financial support by State Grid Corporation of China Science and Technology Projects (SQQHKY0DWJS1800170) and Qinghai Golmud Luneng Energy Co., Ltd Projects "Research on key technologies of Wind-Solar-CSP-Battery storage control system based on Haixi multi-energy complementary integration and optimization demonstration project", is very much appreciated.

\section{References}

1. J. Blunden. Califorlia Energy Commission (2004).

2. R. Sioshansi, P. Denholm. IEEE T SUSTAIN ENERG, 4 , 877-885 (2013).

3. Y. Tianliang, W. Xingquan, L. Zhiwei, L.Xin, W.Kun. PSPC, 45 , 126-132 (2017).

4. M. Shahzad Javed, T. Ma. Energy Procedia, 158 , 6384-6392 (2019).

5. W. Hongbin, C. Bin, G. Caiyun. Transactions of the CSAE, 27, 241-245 (2011).

6. A. Bin, Y. Hongxing, S. Hui, L. Xianbo. ACTA ENERGIAE SOLARIS SINICA, 24 , 540-547 (2003).

7. Y. Yang, S. Guo, D. Liu, R. Lia, Y. Chu. Energy Conversion and Management, 160, 243-250 (2018).

8. C. Yang, Z. Huiquan, Z. Wuzhi, Z. Yuting, Z. Jietan, W. Maochun. Power system technology, 1-8 (2018).

9. M. Petrollese, D. Cocco. Solar Energy, 137 ,477-489 (2016).

10. C. Yang, Y. Zhiwen, Z. Jietan, W. Maochun, Y. Gangui. High Voltage Engineering, 45 , 270-275 (2019).

11. L. Yanping, J. Chunjuan. Proceedings of the CSUEPSA, 27 ,69-74 (2015).

12. X. Chang, Z. Linjuan. Wind farm planning and design[M]. China Water\&Power Press ( 2014).

13. H. Liu, R. Zhai, J. Fu, Y. Wang, Y. Yang. Solar energy, 184 ,391-409 (2019).

14. L. Zhengmao, Z. Feng. Proceedings of the CSEE, 35 , 3569-3576 (2015).
15. Z. Mingzhi, Z. Xiaoming. New Energy Technology, 23-26 (2016). 